\title{
PERFIL COMUNICATIVO DE CRIANÇAS ENTRE 1 E 3 ANOS COM DESENVOLVIMENTO NORMAL DE LINGUAGEM
}

\author{
Communicative profile of children between 1 and 3-years old \\ with normal language development
}

\author{
Mirtes Adiles Sandri (1), Simone Lorelei Meneghetti (2), Erissandra Gomes ${ }^{(3)}$
}

\begin{abstract}
RESUMO
Objetivo: traçar o perfil comunicativo (habilidades comunicativas e interacionais, compreensão verbal e aspectos do desenvolvimento cognitivo) de crianças entre 1 e 3 anos com desenvolvimento normal da linguagem, correlacionando com as variáveis faixa etária e gênero. Métodos: participaram do estudo 24 crianças (12 meninos e 12 meninas), divididas em dois grupos: Grupo 1 (G1) - crianças de 1 e 2 anos e Grupo 2 (G2) - crianças de 2 e 3 anos. O instrumento de avaliação utilizado foi o Protocolo de Observação Comportamental (PROC) (Zorzi e Hage, 2004). Resultados: na análise dos dados quantitativos houve diferença estatisticamente significante quando comparados os grupos por faixa etária $(P<0,05)$. Na comparação dos dados qualitativos pela faixa etária as crianças do $G 2$ foram superiores $(P<0,05)$, exceto no item compreensão da linguagem oral que se mostrou equivalente nos grupos $(P=0,217)$. Em relação ao gênero não houve diferença estatisticamente significante, entretanto observa-se uma superioridade do feminino. Conclusão: o teste utilizado foi sensível para identificar diferenças no desempenho entre os dois grupos etários no que tange ao perfil comunicativo das crianças pesquisadas.
\end{abstract}

DESCRITORES: Desenvolvimento de Linguagem; Testes de Linguagem; Pré-Escolar

\section{INTRODUÇÃO}

A linguagem é a principal forma de expressão que permite a relação entre as pessoas e o seu desenvolvimento depende de uma diversidade de variáveis, como: integridade anatomofisiológica, maturação do Sistema Nervoso Central, aspectos emocionais e sociais, entre outros. Tratando-se de um assunto bastante instigante e abrangente, a linguagem deve ser estudada além das palavras e dos aspectos linguísticos, considerando o seu caráter social e dialógico, ou seja, a habilidade de usá-la

(1) Fonoaudióloga; Associação de Pais e Amigos dos Excepcionais, APAE, São Marcos RS; Especialista em Linguagem pelo CEFAC - Saúde e Educação.

(2) Fonoaudióloga; Associação de Pais e Amigos dos Excepcionais, APAE, Guaporé, RS; Especialista em Linguagem pelo CEFAC - Saúde e Educação.

(3) Fonoaudióloga; Docente do Curso de Fonoaudiologia do Centro Universitário Metodista do Instituto Porto Alegrense e da Universidade Federal de Ciências da Saúde de Porto Alegre, Porto Alegre, RS; Doutora em Ciências Médicas: Pediatria pela Universidade Federal do Rio Grande do Sul. de maneira apropriada aos diferentes contextos e interlocutores, para que ocorra uma comunicação efetiva ${ }^{1-3}$.

Não existe uma teoria que explique satisfatoriamente como a criança adquire a linguagem, pois cada concepção tem um enfoque diferente; porém se faz necessário entender como é sua aquisição, desenvolvimento e organização ${ }^{3}$.

É possível afirmar que, embora haja variabilidade na aquisição da linguagem de cada criança ${ }^{4}$, existe uma sequência cronológica semelhante de desenvolvimento para todas, na qual é possível descrever fases evolutivas e observar um domínio crescente das habilidades cognitivas e comunicativas quando comparadas diferentes faixa etárias ${ }^{1,5}$. Discute-se, também, na literatura, a influência do gênero no processo da aquisição e desenvolvimento da linguagem, embora não haja consenso sobre essa relação ${ }^{6-17}$.

Várias pesquisas já foram e continuam sendo realizadas acerca do desenvolvimento da linguagem ${ }^{2,4,6-31}$, pois é uma habilidade inata do ser humano e somente pelo conhecimento de seu 
funcionamento é que se podem entender os casos em que o seu curso ocorre de forma diferente ou desviante ${ }^{32}$.

Porém, ainda hoje, encontram-se muitas limitações para avaliação da linguagem em crianças pequenas, pois existem poucos instrumentos clínicos à disposição dos fonoaudiólogos, havendo necessidade de mais pesquisas nesta área com características linguísticas do Português falado no Brasil 5,18,19,32. Entre os instrumentos encontrados na literatura brasileira, há o Protocolo de Observação Comportamental (PROC). O PROC é um instrumento pelo qual, através da observação da maneira como a criança explora os objetos e interage com o outro, é possível compreender e analisar o seu desenvolvimento quanto aos aspectos comunicativos, interacionais e cognitivos. $\mathrm{O}$ instrumento em questão é destinado a crianças pequenas e se utiliza de miniaturas de móveis, utensílios domésticos e pessoas ${ }^{5}$.

A partir do exposto acima, dos dados da literatura e da necessidade de aprofundar os conhecimentos sobre o assunto, esta pesquisa teve por objetivo traçar o perfil comunicativo (habilidades comunicativas e interacionais, compreensão verbal e aspectos do desenvolvimento cognitivo) de crianças entre 1 e 3 anos com desenvolvimento normal da linguagem, correlacionando com as variáveis faixa etária e gênero.

\section{MÉTODOS}

Trata-se de um estudo transversal, descritivo e observacional. Participaram da pesquisa 24 crianças entre 1 e 3 anos, com média de idade de 25,29 $\pm 7,49$ meses, frequentadoras das Escolas Municipais de Educação Infantil Amor Perfeito do município São Marcos e Gasparzinho do município de Guaporé, ambas no estado do Rio Grande do Sul. O grupo total de crianças foi estratificado em dois grupos conforme faixa etária e gênero que ficaram assim divididos: grupo 1 (G1) - constituído por doze crianças, seis meninas e seis meninos com idade de 1 e 2 anos e grupo 2 (G2) - constituído por doze crianças, seis meninas e seis meninos com idade de 2 e 3 anos.

O instrumento utilizado foi o $\mathrm{PROC}^{5}$, elaborado para sistematizar observações sobre o comportamento, configurar os níveis evolutivos e modo de funcionamento cognitivo e comunicativo de crianças com idade entre 12 e 48 meses. Os itens que compõem o PROC estão divididos em três áreas: habilidades comunicativas, compreensão da linguagem oral e desenvolvimento cognitivo infantil. Esse instrumento de avaliação apresenta variáveis qualitativas e quantitativas, indicando que a pontuação máxima do teste é de 70 pontos para habilidades comunicativas; 60 pontos para compreensão da linguagem oral; 70 pontos para aspectos do desenvolvimento cognitivo e 200 pontos no escore total.

O procedimento foi organizado para propor uma situação planejada na qual se possa observar e registrar em vídeo a interação da criança com o examinador envolvendo brinquedos pré-selecionados. O material utilizado na pesquisa foi filmadora da marca Panasonic modelo PV-IQ505, fitas VHS-C e brinquedos: bonecos, miniaturas de objetos da casa (mesa, cadeira, cama, privada, sofá, poltrona, geladeira, fogão, pratos, talheres, televisor, pia, xícaras, esponja de limpeza), objetos diversos (pente, escova, seringa de injeção), conjunto de canecas de encaixe com tampas, blocos de madeira, pedaço de tecido e pedaço de papel.

A seleção da amostra respeitou critérios de inclusão e exclusão. Como critério de inclusão foi solicitado aos professores que indicassem as crianças com desenvolvimento normal. Para tal indicação os professores se basearam em um roteiro de observação dos aspectos motores gerais, perceptivos, afetivo-emocionais e da comunicação oral. ${ }^{21}$

Num segundo momento, os pais das crianças indicadas pelos professores foram entrevistados pessoalmente, para coletar dados sobre a história de vida dos sujeitos. Como critérios de exclusão foram considerados: histórico de prematuridade e internações hospitalares frequentes, antecedentes familiares de dificuldade na linguagem ou queixa de atraso/ dificuldade no desenvolvimento foram excluídas da pesquisa.

Após a entrevista com os pais, foi realizada a avaliação que consistiu na filmagem da interação pais/responsável, terapeuta e criança em ambiente extra-escolar durante aproximadamente 45 minutos. Solicitou-se aos pais que brincassem livremente com a criança utilizando os objetos/brinquedos acima descritos e recomendados pelos autores do PROC.

O material filmado foi assistido e analisado em conjunto pelas pesquisadoras que pontuaram o desempenho da criança em cada área seguindo o protocolo do PROC. No caso de dúvida ou divergência em determinado item, as filmagens eram revistas até consenso das pesquisadoras.

Inicialmente foram selecionadas para a pesquisa 36 crianças; cinco foram excluídas respeitando os critérios estabelecidos e três desistiram. No G2 houve número excedente de crianças do sexo feminino, então se realizou sorteio aleatório para seleção; quatro crianças foram excluídas e chegou-se na amostra final da pesquisa.

As instituições participantes assinaram autorização para realização da pesquisa e os pais ou 
responsáveis o Termo de Consentimento Livre e Esclarecido baseado na Resolução 196/96. Este projeto foi aprovado pelo Comitê de Ética em Pesquisa do CEFAC sob o número 137/06.

Para a análise estatística foi utilizado o software Statistical Package for Social Science (SPSS) 10.0 for Windows. Todos os testes foram realizados na forma bi-caudal, admitindo-se como estatisticamente significativos os valores de $\mathrm{P}$ menores que 0,05 . A análise descritiva das variáveis quantitativas foi realizada por meio da observação do cálculo de médias e desvio-padrão, bem como de mediana e intervalo interquartil. Para as variáveis qualitativas, foi calculada a frequência absoluta. Comparando os gêneros e os grupos etários (G1 e G2) aplicaram-se os testes de Mann-Whitney, qui-quadrado e exato de Fisher. Utilizou-se o Teste de Mann-Whitney para testar os resultados das variáveis quantitativas, sem distribuição normal, entre os gêneros e os grupos etários; o Teste de Qui-quadrado na análise de dados qualitativos das variáveis entre os gêneros e os grupos etários e o Teste exato de Fisher na análise de dados qualitativos das variáveis, quando estas eram dicotômicas, entre os gêneros e os grupos etários.

\section{RESULTADOS}

O total dos dados quantitativos descritivos da amostra referentes ao PROC foi de 55 dos 70 pontos esperados para habilidades comunicativas, de 50 dos 60 pontos esperados para compreensão da linguagem oral e de 42 dos 70 pontos esperados para desenvolvimento cognitivo. O escore total foi de 147 pontos num total de 200.

Os dados gerais das variáveis quantitativas, divididos pelos grupos etários, estão apresentados na Tabela 1 e mostram que há diferença estatisticamente significante entre crianças de 1 e 2 anos e de 2 e 3 anos, no que se refere às habilidades comunicativas, compreensão da linguagem oral e desenvolvimento cognitivo.

Optou-se, também, por estratificar a amostra pelo gênero, sem considerar a faixa etária (Tabela 2). Entretanto nenhuma variável apresentou diferença estatisticamente significante $(P<0,05)$.

O PROC, além dos dados quantitativos, faz uma análise qualitativa. A análise total dos descritores qualitativos referentes à habilidade comunicativa, organização linguística, compreensão linguagem oral e características gerais do desenvolvimento cognitivo são apresentados na Tabela 3. Os dados dessa tabela mostram que a maioria das crianças apresentou comunicação intencional plurifuncional produzindo enunciados, com ampla participação em atividade dialógica, compreensão ligada ao contexto imediato e desenvolvimento cognitivo no nível representativo.

Os resultados obtidos na análise qualitativa de cada grupo etário G1 e G2 são apresentados na Tabela 4. Os grupos G1 e G2 diferiram estatisticamente nas habilidades comunicativas, na organização linguística e no desenvolvimento cognitivo $(P<0,05)$, entretanto não houve diferença estatística na compreensão da linguagem.

Tabela 1 - Comparação, entre os grupos etários, das variáveis quantitativas

\begin{tabular}{lccccc}
\hline Variável & & $\mathbf{G 1}$ & & $\mathbf{G}$ & \\
& & $\mathbf{n}=\mathbf{1 2}$ & & Valor $\mathbf{P}$ \\
\hline total habilidades comunicativas & 43 & $(33$ a 54) & 61 & $(56$ a 67) & $<0,0001$ \\
total compreensão linguagem oral & 40 & $(40$ a 47) & 50 & $(50$ a 57) & 0,001 \\
total desenvolvimento cognitivo & 19 & $(11$ a 33) & 50 & $(47$ a 53) & $<0,0001$ \\
total geral & $101(86$ a 137) & $162(155$ a 176) & $<0,0001$ \\
\hline
\end{tabular}

Mediana (intervalo interquartil), Mann-Whitney; $\mathrm{P}<0,05$

Tabela 2 - Comparação, entre os gêneros, das variáveis quantitativas

\begin{tabular}{|c|c|c|c|}
\hline Variável & $\begin{array}{c}\text { Masculino } \\
\mathrm{n}=12\end{array}$ & $\begin{array}{c}\text { Feminino } \\
n=12\end{array}$ & Valor $\mathbf{P}$ \\
\hline total habilidades comunicativas & (35 a 60) & (46 a 63) & 0,443 \\
\hline total compreensão linguagem oral & $(40$ a 50$)$ & $(42$ a 50$)$ & 0,443 \\
\hline total desenvolvimento cognitivo & 42 (11 a 50) & $42 \quad(28$ a 50$)$ & 0,551 \\
\hline total geral & $141(87$ a 160$)$ & $152(109$ a 164$)$ & 0,410 \\
\hline
\end{tabular}

Mediana (intervalo interquartil), $\mathrm{P}<0,05$, Mann-Whitney 


\begin{tabular}{lc}
\hline Variável & Descritor \\
\hline $\begin{array}{l}\text { habilidade comunicativa } \\
\text { habilidade comunicativa comunicação intencional plurifuncional ampla } \\
\text { participação atividade dialógica meio não simbólico/não verbal } \\
\text { habilidade comunicativa comunicação intencional plurifuncional ampla } \\
\text { participação atividade dialógica meio verbal contexto imediato } \\
\text { habilidade comunicativa comunicação intencional plurifuncional ampla } \\
\text { participação atividade dialógica meio verbal contexto não imediato }\end{array}$ & $6(25 \%)$ \\
organização linguística & $13(54 \%)$ \\
não apresenta organização linguística & $5(21 \%)$ \\
produção palavras isoladas & \\
produção enunciados & $5(21 \%)$ \\
produção discurso & $2(8 \%)$ \\
compreensão linguagem oral & $11(46 \%)$ \\
compreende ordens até duas ações, ligadas contexto imediato & $6(25 \%)$ \\
compreende ordens com três ou mais ações, não ligadas contexto imediato & \\
características gerais do desenvolvimento cognitivo & $21(88 \%)$ \\
sensório motor fases avançadas & $3(12 \%)$ \\
transição entre sensório motor e representativo & \\
representativo & $7(29 \%)$ \\
\hline
\end{tabular}

$\mathrm{n}=24$, número absoluto (frequência)

Tabela 4 - Comparação, entre os grupos etários, das variáveis qualitativas

\begin{tabular}{|c|c|c|c|}
\hline Variável & $\begin{array}{c}\mathrm{G1} \\
\mathrm{n}=12\end{array}$ & $\begin{array}{c}\mathrm{G} 2 \\
\mathrm{n}=12\end{array}$ & Valor P \\
\hline \multicolumn{4}{|l|}{ habilidade comunicativa } \\
\hline $\begin{array}{l}\text { habilidade comunicativa comunicação intencional } \\
\text { plurifuncional ampla participação atividade dialógica } \\
\text { meio não simbólico/não verbal }\end{array}$ & $6(50 \%)$ & $0(0 \%)$ & $0,004^{1}$ \\
\hline $\begin{array}{l}\text { habilidade comunicativa comunicação intencional } \\
\text { plurifuncional ampla participação atividade dialógica } \\
\text { meio verbal contexto imediato }\end{array}$ & $6(50 \%)$ & 7 (58\%) & \\
\hline $\begin{array}{l}\text { habilidade comunicativa comunicação intencional } \\
\text { plurifuncional ampla participação atividade dialógica } \\
\text { meio verbal contexto não imediato }\end{array}$ & $0(0 \%)$ & $5(42 \%)$ & \\
\hline \multicolumn{3}{|l|}{ organização linguística } & $0,004^{1}$ \\
\hline não apresenta organização linguística & $5(42 \%)$ & $0(0 \%)$ & \\
\hline produção palavras isoladas & $2(16 \%)$ & $0(0 \%)$ & \\
\hline produção enunciados & $5(42 \%)$ & $6(50 \%)$ & \\
\hline produção discurso & $0(0 \%)$ & $6(50 \%)$ & \\
\hline \multicolumn{3}{|l|}{ compreensão linguagem oral } & $0,217^{2}$ \\
\hline $\begin{array}{l}\text { compreende ordens até duas ações, ligadas contexto } \\
\text { imediato }\end{array}$ & $12(100 \%)$ & $9(75 \%)$ & \\
\hline $\begin{array}{l}\text { compreende ordens com três ou mais ações, não } \\
\text { ligadas contexto imediato }\end{array}$ & $0(0 \%)$ & $3(25 \%)$ & \\
\hline \multicolumn{3}{|l|}{ características gerais do desenvolvimento cognitivo } & $<0,0001^{1}$ \\
\hline sensório motor fases avançadas & $7(58 \%)$ & $0(0 \%)$ & \\
\hline transição entre sensório motor e representativo & $5(42 \%)$ & $1(8 \%)$ & \\
\hline representativo & $0(0 \%)$ & $11(92 \%)$ & \\
\hline
\end{tabular}

Mediana (intervalo interquartil), Teste de qui-quadrado', Teste exato de Fisher ${ }^{2}, \mathrm{P}<0,05$ 
Observou-se que as crianças do G1 estão entre o meio não verbal e não simbólico e meio verbal ligado ao contexto imediato, enquanto as crianças do G2 estão em nível mais evoluído entre o meio verbal ligado ao contexto imediato e não ligado ao contexto imediato. A organização linguística das crianças do G2 é superior, quando comparadas ao G1. Quanto à compreensão da linguagem oral observou-se que tanto as crianças de ambos grupos atendem a ordens ligadas ao contexto imediato, porém no G2, já aparecem respostas a ordens não ligadas ao contexto imediato. Quanto ao desenvolvimento cognitivo as crianças do $\mathrm{G} 1$ encontram-se no nível sensório motor avançado ou em transição para o representativo. Já as do G2 estão quase na sua totalidade no nível representativo.

A análise comparando os sexos (Tabela 5) apresentou valores sem relevância significativa, porém observou-se que as meninas falam mais cedo e produzem mais discurso.

Tabela 5 - Comparação, entre os gêneros, das variáveis qualitativas

\begin{tabular}{|c|c|c|c|}
\hline Variável & $\begin{array}{c}\text { Masculino } \\
\mathrm{n}=12\end{array}$ & $\begin{array}{c}\text { Feminino } \\
\mathrm{n}=12\end{array}$ & Valor $\mathbf{P}$ \\
\hline habilidade comunicativa & & & $0,624^{1}$ \\
\hline $\begin{array}{l}\text { habilidade comunicativa comunicação intencional } \\
\text { plurifuncional ampla participação atividade dialógica } \\
\text { meio não simbólico/não verbal }\end{array}$ & $4(33 \%)$ & $2(17 \%)$ & \\
\hline $\begin{array}{l}\text { habilidade comunicativa comunicação intencional } \\
\text { plurifuncional ampla participação atividade dialógica } \\
\text { meio verbal contexto imediato }\end{array}$ & $6(50 \%)$ & $7(59 \%)$ & \\
\hline $\begin{array}{l}\text { habilidade comunicativa comunicação intencional } \\
\text { plurifuncional ampla participação atividade dialógica } \\
\text { meio verbal contexto não imediato }\end{array}$ & $2(17 \%)$ & $3(25 \%)$ & \\
\hline organização liuguística & & & $0,207^{1}$ \\
\hline não apresenta organização linguística & $4(33 \%)$ & $1(8 \%)$ & \\
\hline produção palavras isoladas & $0(0 \%)$ & $2(17 \%)$ & \\
\hline produção enunciados & $6(50 \%)$ & $5(42 \%)$ & \\
\hline produção discurso & $2(17 \%)$ & $4(33 \%)$ & \\
\hline compreensão linguagem oral & & & $0,999^{2}$ \\
\hline $\begin{array}{l}\text { compreende ordens até duas ações, ligadas contexto } \\
\text { imediato }\end{array}$ & $10(83 \%)$ & $11(92 \%)$ & \\
\hline $\begin{array}{l}\text { compreende ordens com três ou mais ações, não } \\
\text { ligadas contexto imediato }\end{array}$ & $2(17 \%)$ & $1(8 \%)$ & \\
\hline características gerais do desenvolvimento cognitivo & & & $0,347^{2}$ \\
\hline sensório motor fases avançadas & $5(42 \%)$ & $2(17 \%)$ & \\
\hline transição entre sensório motor e representativo & $2(16 \%)$ & $4(33 \%)$ & \\
\hline representativo & $5(42 \%)$ & $6(50 \%)$ & \\
\hline
\end{tabular}

Teste de qui-quadrado ${ }^{1}$, Teste exato de Fisher ${ }^{2}$

\section{DISCUSSÃO}

Os dados quantitativos do total da amostra não são discutidos em relação ao seu escore (pontuação alcançada), pois não foram encontradas referências na literatura relacionadas a pesquisas com o PROC; assim, apenas se indica os achados que poderão servir de referência para futuras pesquisas.

$\mathrm{Na}$ análise dos resultados da Tabela 1 , ao serem comparados G1 e G2, verificou-se que o G2 teve melhor desempenho em todas as áreas: habilidades comunicativas, compreensão oral e desenvolvimento cognitivo e que a diferença entre os grupos foi estatisticamente significativa. Autores concluem em seus estudos que o desenvolvimento infantil segue uma ordem cronológica evolutiva nas quais as habilidades conversacionais vão se aprimorando, utilizando mais turnos, ampliando o vocabulário, aumentando o grau de complexidade na linguagem, nas brincadeiras e aproximando-se do modelo adulto 15-17,20-25.

Estudos relatam haver diferença entre gêneros, no desenvolvimento da linguagem, constatando que as meninas têm um desempenho levemente melhor que os meninos, apesar das correlações não terem apontado significância estatística ${ }^{6-9}$. Esses achados 
corroboram os encontrados no presente estudo e apresentados na Tabela 2. No entanto algumas pesquisas divergem a respeito, mostrando que as meninas são superiores em relação às habilidades comunicativas e vocabulário 10-17.

Analisando os resultados da Tabela 3 e comparando com dados de outra pesquisa ${ }^{15}$ verificou-se que as crianças com desenvolvimento adequado adquirem linguagem oral no segundo ano de vida, e que esse período é um tempo de rápidas aquisições quando a criança passa da produção de palavra-frase para sentenças gramaticais complexas. Porém a linguagem e a compreensão ainda estão relacionadas à sua ação, ou seja, ligadas ao contexto específico em que são proferidas ${ }^{20-26}$. Quanto ao desenvolvimento cognitivo a maioria das crianças está no período representativo considerando este a partir das condutas pré-simbólicas. Estudos mostram que crianças com desenvolvimento normal com o passar do tempo tendem a desenvolver uma brincadeira mais complexa e simbólica ${ }^{24-27}$.

Outro estudo realizado com crianças de 20 meses para pesquisar o desenvolvimento da brincadeira e da linguagem concluiu que com a mãe, a brincadeira tende a ser mais duradoura e com maior grau de simbolismo ${ }^{28}$. Dessa forma esse fator também pode ter interferido para um bom desempenho das crianças em nosso estudo.

Em um estudo realizado com 15 crianças para analisar o desenvolvimento da linguagem do nível pré-linguístico até a construção de frases verificouse que na fase pré-linguística e de palavra-frase os meios de comunicação predominantes foram gestual e vocal ao passo que na fase de múltiplas palavras o meio predominante foi o verbal ${ }^{15}$. O mesmo aconteceu na presente pesquisa na análise das variáveis qualitativas dos grupos etários (Tabela 4) onde se observou que as crianças do G1 estão entre o meio não verbal e não simbólico e meio verbal ligado ao contexto imediato, enquanto que as crianças do G2 estão em um nível mais evoluído entre o meio verbal ligado ao contexto imediato e não ligado ao contexto imediato. Conclui-se que aparecem crianças dos dois grupos no nível meio verbal ligado ao contexto imediato por ser esse um período de transição no qual as palavras estão dependentes do que está no campo de percepção da criança e a fatos recém finalizados ou que acontecerão em seguida ${ }^{20}$.

Ainda, discutindo a Tabela 4, os achados deste estudo estão de acordo com outros realizados em crianças nessa faixa etária. Observa-se que no primeiro ano de vida as crianças têm condições de usar as primeiras palavras, sendo a linguagem caracterizada por enunciados de uma só palavra; dos 18 aos 24 meses surgem enunciados de dois elementos, formando um esboço de frase e a partir dos 24 meses a criança inicia e mantém a conversação por turnos curtos ${ }^{1,5,29}$.

Quanto à compreensão da linguagem oral observou-se que tanto as crianças do G1 quanto às do G2 atendem a ordens ligadas ao contexto imediato. Esse resultado corrobora pesquisa que afirma que a criança atende a pedidos desde muito cedo, guiada por pistas contextuais, pelo conhecimento da situação em que se encontra e pela informação linguística ${ }^{30}$. No entanto, no G2, já aparecem respostas a ordens não ligadas ao contexto imediato o que demonstra evolução nesse aspecto do desenvolvimento. Esses achados vão ao encontro aos dados obtidos na literatura que afirmam que por volta dos dois anos e seis meses a crianças já compreende ordens não-ligadas ao contexto imediato ${ }^{20,26}$. Outro estudo comparando crianças de 2 anos e 6 meses e crianças de 3 anos mostrou que as maiores necessitam de menos instruções para resolverem corretamente uma tarefa dada ${ }^{27}$.

Estudo realizado com 71 crianças entre 2 e 6 anos com desenvolvimento normal de linguagem e com alteração no desenvolvimento de linguagem para identificar os tipos de brincadeiras, parceiros e brinquedos utilizados mostrou que dos 12 ao 36 meses o jogo fica mais complexo, generalizado e simbólico e a criança torna-se apta a sustentar jogos na ausência de materiais ou objetos concretos. Tais achados vêm ao encontro da presente pesquisa que demonstra que as crianças do G1 encontramse no nível sensório motor avançado ou em transição para o representativo e as do G2 estão quase na sua totalidade no nível representativo e também confirma estudo anterior que constata o surgimento do simbolismo como um marco entre o período sensório motor e representativo ${ }^{31}$. $\mathrm{O}$ aparecimento destas condutas simbólicas no segundo ano de vida da criança serve como referencial de um bom desenvolvimento ${ }^{1}$.

A análise comparando os gêneros (Tabela 5) mostrou que nas habilidades comunicativas e na organização linguística as meninas falam mais cedo e produzem mais discurso, porém valores sem relevância significativa, o que corrobora os dados encontrados em pesquisas nacionais e internacionais já citadas anteriormente ${ }^{6-9}$.

\section{CONCLUSÃO}

Comparando os grupos G1 e G2 se observou que as crianças do $\mathrm{G} 2$, de faixa etária maior, tiveram melhor desempenho em todas as áreas. Não houve diferença estatisticamente significante na análise comparativa entre os gêneros. 
A respeito do instrumento de avaliação utilizado conclui-se que embora ainda não possua dados de validação, foi sensível para identificar diferenças no desempenho entre os dois grupos etários. Por ser um teste nacional, estaria ajustado à nossa realidade e a sua utilização pelos profissionais permitiria avaliar e abordar o processo comunicativo de forma ampla, realizando diagnóstico mais efetivo e favorecendo assim o planejamento das intervenções terapêuticas.

\begin{abstract}
Purpose: to trace the communicative profile (communicative and interactive abilities, verbal comprehension and cognitive development aspects) of children with normal linguistic development between 1 and 3-year old, correlating with the variables of age groups and gender. Methods: twentyfour children (12 boys and 12 girls) took part in the study, divided in two groups: Group 1 (G1) children between 1 and 2-year old, and Group 2 (G2) -children between 2 and 3-year old. The used protocol was Behavioral Observation Protocol ("Protocolo de Observação Comportamental - PROC") (Zorzi and Hage, 2004). Results: there was a statistically significant difference in the quantitative data when the groups where compared by their age $(P<0.05)$. When comparing qualitative data by their age group, children of the $\mathrm{G} 2$ were better $(P<0.05)$, except in the item with regards to the oral language understanding, which resulted being equivalent between the groups ( $P=0.217)$. There was no significant statistical difference related to gender, however, we observed superiority in female gender. Conclusion: the used test was sensitive for identifying the differences in performance between the two age groups, with regards to the communicative profile of the studied children.
\end{abstract}

KEYWORDS: Language Development; Language Tests; Child, Preschool

\section{REFERÊNCIAS}

1. Zorzi JL. Aspectos básicos para compreensão, diagnóstico e prevenção dos distúrbios de linguagem na infância. Rev CEFAC. 2000; 2(1):11-5.

2. Befi-Lopes DM, Rodrigues A, Rocha LC. Habilidades linguístico-pragmáticas em crianças normais e com alteração de desenvolvimento de linguagem. Pró-Fono. 2004; 16(1):57-66.

3. Acosta V, Moreno A, Ramos V, Quintana A, Espino O. Avaliação da linguagem: teoria e prática do processo de avaliação infantil do comportamento linguístico infantil. São Paulo: Santos; 2003.

4. Lamprecht RR. A aquisição da fonologia do português na faixa etária dos 2:9 - 5:5. Letras de Hoje. 1993; 28(2):99-106.

5. Zorzi JL, Hage SRV. PROC Protocolo de observação: avaliação de linguagem e aspectos cognitivos infantis. São José dos Campos: Pulso; 2004.

6. Rezende MA, Beteli VC, Santos JLF. Avaliação de habilidades de linguagem e pessoal-sociais no teste Denver II em instituições de educação infantil. Acta Paul Enferm. 2005; 18(1):56-63.

7. Lejarraga $H$, Del Pino $M$, Kelmansky $D$, Laurencena E, Ledri LI, Laspiur M, et al. Edad de la pauta madurativa "mamá-papá específico", en una muestra de niños sanos. Arch Argent Pediatr. 2005; 103(6):514-8.

8. Befi-Lopes DM, Cáceres AM, Araújo K. Aquisição de verbos em pré-escolares falantes do português brasileiro de linguagem na infância. Rev CEFAC. 2007; 9(4):444-52.

9. Braz FS, Salomão NMR. A fala dirigida a meninos e meninas: um estudo sobre o input materno e suas variações. Psicologia: Reflexão e Crítica. 2002; 15(2):333-44 .

10. Fensterseifer A, Ramos APF. Extensão média de enunciados em crianças de 1 a 5 anos. PróFono. 2003; 15(3):251-8.

11. Durmazlar N. Turkish children's performance on Denver II: effect of sex and mother's education. Dev Med Child Neurol. 1998; 40(6):411-6.

12. Normand MT, Parisse $C$, Cohen H. Lexical diversity and productivity in French preschooolers developmental and biosocial aspects by developmental, gender and sociocultural factors. Clin Linguist Phon. 2008; 22(1):47-58.

13. Reilly S, Wake M, Bavin EL, Prior M, Williams J, Bretherton L, Eadie P, Barrett Y, Ukoumunne OC. Predicting language at 2 years of age: a prospective community study. Pediatrics. 2007; 120(6):1441-9.

14. Bornstein MC, Leach DB, Haynes M. Vocabulary competence in first- and secondborn siblings of 
the same chronological age. J Child Lang. 2004; 31:855-73.

15. Leaper C, Smith TE. A meta-analytic review of gender variations in children's language use: talkativeness, affiliative speech, and assertive speech. Developmental Psychology. 2004; 40(6): 993-1027.

16. Stennes LM, Burch MM, Sen MG, Bauer PJ. A longitudinal study of gendered vocabulary and communicative action in young children. Developmental Psychology. 2005; 41(1):75-88.

17. Pedromônico MRM, Affonso LA, Sanudo A. Vocabulário expressivo de crianças entre 22 e 36 meses: estudo exploratório. Rev. Bras. Cresc. Desenv. Hum. 2002; 12(2):13-22.

18. Giusti E, Befi-Lopes DM. Performance de sujeitos falantes do Português e do Inglês no Test of Early Language Development. Pró-Fono. 2008; 20(1):13-18.

19. Chiari BM, Bragatto EL, Barbosa T, Strobilius $\mathrm{R}$, Soares TCB. Avaliação da intencionalidade e funcionalidade da comunicação em crianças deficientes auditivas e ouvintes entre 24 e 60 meses. Pró-Fono. 2002; 14(2):187-198.

20. Puglisi ML, Befi-Lopes DM, Takiuchi N. Utilização e compreensão de preposições por crianças com distúrbio específico de linguagem. Pró-Fono. 2005; 17(3):331-44.

21. Rocha LC, Befi-Lopes DM. Analyses of answers presented by children with and without specific language impairment (original title: Análise pragmática das respostas de crianças com e sem distúrbio específico de linguagem). Pró-Fono. 2006; 18(3):229-38.

22. Basílio CS, Puccini RF, Silva EMK, Pedromônico RM. Living conditions and receptive vocabulary of children aged two to five years. Rev Saúde Pública. 2005; 39(5):725-30.

23. Resegue, MM, Sedano D, Pacheco E, Hage SRV. Como conversam as crianças de 03 anos. In: V Congresso Internacional de Fonoaudiologia; 2003 set; Fortaleza, Ceará.
24. Befi-Lopes DM, Elmôr L de RM. Experimentação de um checklist no desenvolvimento das brincadeiras em crianças com alteração no desenvolvimento da linguagem. J Bras de Fonoaudiol 2006; 6(24): 26-36.

25. Wetherby AM, Cain DH, Yonclas DG, Walker VG. Analysis of intentional communication of normal children from the prelinguistic to the multiword stage. Journal of Speech and Hearing Research. 1988; 31 : 240-52.

26. Mantovani DA, Zorzi JL, Hage SRV. Comparação das habilidades comunicativas e de compreensão de crianças com alterações no desenvolvimento da linguagem. [CD Rom]. In: XIII Congresso Brasileiro de Fonoaudiologia; 2005 set 28-30; Santos. Revista da Sociedade Brasileira de Fonoaudiologia suplemento especial ISSN1807-3115.

27. Salsa AM. Desarrollo simbólico em niños pequeños: El rol de la instrucción em la comprensión y el uso de símbolos. Interdisciplinaria. 2004; (21)1: 4-27.

28. Mendes DMLF, Moura MLS. Desenvolvimento da brincadeira e linguagem em bebês de 20 meses. Psicologia: Teoria e Pesquisa. 2004; 20(3):215-22. 29. Bornstein MH, Tamis-LeMonda CS. Maternal responsiveness and infant mental abilities: specific predictive relations. Infant Behavior and Development.1997; 20(3):283-96.

30. Bohn CBA Compreensão de diretivos por crianças [dissertação] Porto Alegre (RS): Universidade Federal do Rio Grande do Sul; 2005.

31. Novaes APDC. A importância do jogo e do brincar em terapia fonoaudiológica. Rev CEFAC. 2000; 2(2):45-54.

32. Ribas ML, Kautzmann MC, Ribeiro R. Aquisição sintática: revisão bibliográfica. In: Anuário de Fonoaudiologia Feevale. Novo Hamburgo: Feevale; 2003. p.87-96.

33. Pereira DL, Santos AMS, Osborn E. Ação preventiva na escola: aspectos relacionados com a integração professor e aluno e a comunicação humana. In: Viera RM, Vieira MM, Ávila CRB, Pereira LD. Fonoaudiologia e saúde pública. 2. ed. Carapicuíba: Pró-Fono; 2004.

RECEBIDO EM: 09/11/2007

ACEITO EM: 04/02/2009

Endereço para correspondência:

Mirtes Adiles Sandri

Av. Venâncio Aires, 660 sala 81

São Marcos - RS

CEP: 95190-000

E-mail: mirtessandri@yahoo.com.br 